\section{FRI0352 EFFICACY OF BIOLOGICS IN PATIENTS WITH REFRACTORY TAKAYASU ARTERITIS AND ANALYSIS OF THEIR GENETIC BACKGROUNDS}

Y. Gon ${ }^{1}$, H. Yoshifuji ${ }^{1}$, T. Nakajima ${ }^{1}$, K. Murakami ${ }^{1}$, R. Nakashima ${ }^{1}$, K. Ohmura ${ }^{1}$, C. Terao ${ }^{2}$, T. Mimori ${ }^{1} .{ }^{1}$ Department of Rheumatology and Clinical Immunology, Graduate School of Medicine, Kyoto University; ${ }^{2}$ Center for Genomic Medicine, Kyoto University, Kyoto, Japan

Background: Takayasu arteritis (TAK) is a rare disease that mostly affects young females and causes inflammation, stenosis and dilatation of the aorta and its main branches. Although glucocorticoids are effective, relapses occur frequently [1]. Recently, the efficacy of biologics has been reported in refractory TAK. HLA-B*52 is a major genetic risk factor and associated with the severity [2]. Our group reported a SNP in the regions of IL12B gene associated with the onset of TAK, and the patients with risk alleles at the SNP showed significantly severer symptoms [3].

Objectives: To evaluate the efficacy and safety of biologics in the treatment of refractory TAK, we analyzed the clinical profiles and genetic information of patients treated with biologics in our institute.

Methods: We searched for TAK patients who used biologics in 163 TAK cases treated in Kyoto University Hospital from 2000 to 2016. From medical records, we extracted information of classifications, symptoms, complications, ESR, CRP, dose of prednisolone (PSL), use of immunosuppressive agents (IS), and adverse events at 0,1,612 months after the initiation of biologics.

Results: Of 163 cases, 12 (7.4\%) were treated with biologics. Two were excluded because biologics were used for coexisting rheumatoid arthritis. Remaining 10 cases were treated with infliximab (IFX, $3-5 \mathrm{mg} / \mathrm{kg}$ div. bimonthly, $\mathrm{N}=4$ ), tocilizumab (TCZ, $8 \mathrm{mg} / \mathrm{kg}$ div. monthly, $\mathrm{N}=3$ ) or ustekinumab (UST, $45 \mathrm{mg}$ s.c. trimonthly, $\mathrm{N}=3$ ). Sex ratio was 1:9. The age of onset was $29.4 \pm 12.2$ y.o. (mean \pm S.D.). According to Hata's classification [4], they were categorized into types I (N=3), Ila (2) and V (5). Three patients had aortic regurgitation. The number of IS used in the past was $1.7 \pm 0.8$ per patient. $B^{*} 52$ was positive in $7(70 \%)$ patients. All the cases had risk-type alleles (A vs. C) of IL12B SNP (AA: $A C$ : $C C=4: 6: 0)$. ESR was significantly decreased $(p=0.009)$ and $C R P$ showed a tendency to decrease after 12 mo. (Table 1). Dose of PSL was significantly decreased from $10.6 \pm 1.9$ to $7.7 \pm 2.4 \mathrm{mg} /$ day after $12 \mathrm{mo}$. $(p=0.018)$. We could not find significant improvements in imaging modalities such as MR and ultrasonography. Overall duration of treatments with biologics was $39 \pm 22$ months. Two cases discontinued IFX due to occurrence of breast cancer and infusion reaction, respectively. Chronic heart failure was exacerbated in a case treated with TCZ. Two of 3 patients treated with UST exhibited steroid- sparing effects and none of them discontinued UST in the mean observation period of $31 \mathrm{mo}$.

Table 1. Changes after the treatment with biologics

\begin{tabular}{lccccc}
\hline & Before & $1 \mathrm{mo}$. & $6 \mathrm{mo}$. & $12 \mathrm{mo}$. & $P$ (before vs. 12 mo.) \\
\hline ESR $(\mathrm{mm} / \mathrm{h})$ & $31 \pm 16$ & $14 \pm 9$ & $14 \pm 12$ & $12 \pm 12$ & 0.009 \\
$\mathrm{CRP}(\mathrm{mg} / \mathrm{L})$ & $15 \pm 19$ & $8 \pm 13$ & $4 \pm 5$ & $2 \pm 4$ & 0.075 \\
PSL $(\mathrm{mg} /$ day) & $10.6 \pm 1.9$ & $10.5 \pm 2.0$ & $9.0 \pm 2.3$ & $7.7 \pm 2.4$ & 0.018 \\
\hline
\end{tabular}

Conclusions: Biologics showed steroid-sparing effects in refractory TAK. Besides, there was a tendency that patients who used biologics had $B^{*} 52$ and the risk-type allele of IL12B SNP, implicating the refractory courses of them.

References:

[1] Maksimowicz-McKinnon K, Clin Exp Rheumatol 25:S58-9, 2007.

[2] Numano F, Jpn Circ J 46(2):184-9, 1982.

[3] Terao C, Am J Hum Genet 93(2):289-97, 2013.

[4] Hata A, Int J Cardiol 54 Suppl:S155-163, 1996.

Disclosure of Interest: None declared

DOI: 10.1136/annrheumdis-2017-eular.4533

\section{FRIDAY, 16 JUNE 2017}

\section{Scleroderma, myositis and related syndromes - etiology, pathogenesis and animal models}

\section{FRI0353 SERUM PROTEOMICS PROFILE IN SYSTEMIC SCLEROSIS PATIENTS}

M. De Santis ${ }^{1}$, E. Generali ${ }^{1}$, A. Ceribelli ${ }^{1}$, N. Isailovic ${ }^{1}$, M. Caprioli ${ }^{1}$, G.M. Guidelli ${ }^{1}$, C. Selmi ${ }^{1,2},{ }^{1}$ Rheumatology and Clinical Immunology, Humanitas Research Hospital, Rozzano; ${ }^{2}$ BIOMETRA Department, University of Milan, Milan, Italy

Background: Systemic sclerosis (SSc) is characterized in virtually all patients by anti-nuclear antibodies (ANA) with anti-centromere (ACA), anti-topoisomerase I (ant-Scl70), and anti-RNA polymerase III antibodies identifying patient subgroups. However, there are no reliable biomarkers predicting SSc susceptibility and internal organ involvement.

Objectives: We aimed to identify serum protein biomarkers associated with SSc and interstitial lung disease (ILD), using a highly multiplexed proteomic technology.

Methods: We analyzed serum samples of 3 patients with SSc and ILD (2 limited
SSc: one ANA positive and one anti-Scl70 positive, and one diffuse SSc anti-Scl70 positive), 3 patients with SSC and no ILD (ACA positive limited SSC), and 4 healthy controls. All subjects were women and age matched. Serum proteomics profiling was performed using the SOMAscan platform (SomaLogic, Inc., Boulder, CO, USA). Statistical analysis included Student's t-test and were performed using the SomaSuite software (SomaLogic, Boulder, CO, USA).

Results: Proteomic analysis identified 33 proteins which differentiate SSc from HC and 9 proteins which differentiate SSc patients with and without ILD. Compared to healthy controls, SSc cases showed an altered expression of proteins involved in extracellular matrix formation and cell-cell adhesion, angiogenesis, and lymphocyte recruitment, activation, and signaling, while an overall inhibition of neutrophil function was noted. An interferon and IL-1 signatures were also found. Patients with SSc and ILD manifested increased protein levels related to intracellular signaling and cell cycle, along with an increase of monocyte chemoattractants and ligands for the leukocyte adhesion compared to SSc without ILD. We further observed a decrease in B cell stimulating factor and IL-22 signaling in SSc with ILD.

Conclusions: Serum proteomic profiles can differentiate SSc from healthy controls and SSc patients with and without ILD; moreover, our results identify biomarkers with a putative pathogenetic significance.

Disclosure of Interest: None declared

DOI: 10.1136/annrheumdis-2017-eular.5693

\section{FRI0354 CIRCULATING MICROPARTICLE SUBSETS ARE ASSOCIATED WITH PATIENTS WITH EXTENDED FIBROTIC PHENOTYPE IN SYSTEMIC SCLEROSIS}

K. Sakata ${ }^{1}$, H. Yasuoka ${ }^{1}$, M. Koseki ${ }^{1}$, K. Yoshimoto ${ }^{1}$, M. Kuwana ${ }^{2}$,

T. Takeuchi ${ }^{1} .{ }^{1}$ Division of Rheumatology, Department of Internal Medicine, Keio University School of Medicine; ${ }^{2}$ Department of Allergy and Rheumatology, Nippon Medical School Graduate School of Medicine, Tokyo, Japan

Background: In systemic sclerosis (SSc), systemic remodeling, microvascular injuries and production of autoantibodies are well known as disease hallmarks However, the master regulator of these characteristics is still unclear. Microparticle (MP) is a small membrane vesicle, released from various kinds of cells, and distributes systemically through circulation ${ }^{1}$. Recent reports revealed that MP contains various growth factors, proteases and cytokines including TGF- $b^{2}$, suggesting that MP can contribute to immune and mesenchymal responses as a conveyor ${ }^{3)}$. and involve with disease process of SSc. Although, the possible involvement of MPS in the pathogenesis of SSc is indicated, the detailed association of MPs including MP subsets with SSc is not well clarified.

Objectives: To elucidate the association between circulating MP subsets and patients' characteristic of SSc.

Methods: Thirty-six patients with SSc and 13 healthy controls were involved in this study. Platelet-rich plasma containing microparticles was isolated from whole blood using gradient centrifugation and analyzed using flow cytometer. MP was defined as particles smaller than $1.0 \mu \mathrm{m}$ diameter using Megamix ${ }^{\circledR}$, and identified each MP subsets based on expression of cell type-specific surface markers; platelet $\left(\mathrm{CD} 31^{+} \mathrm{CD} 41^{+}\right)$, endothelial cell $\left(\mathrm{CD} 31^{+} \mathrm{CD} 41^{-}\right)$and monocyte $\left(C D 14^{+} C D 45^{+}\right)$using fluorescence-tagged antibodies. Clinical information was retrospectively collected from patients' records, and the correlation with the number of each MP subset was analyzed.

Results: Mean age of 36 patients with SSc was $60.9 \pm 1.8$ years, $94 \%$ was female, and mean disease duration was $11.2 \pm 9.5$ years. Patients with diffuse cutaneous SSc (dcSSc) was $6(17 \%)$. As for the proportion of MP subsets, platelet-derived MP (PMP) was the largest subset, followed by endothelial cell-derived MP (EMP) and monocyte-derived MP (MoMP) in all samples. In patients with SSc, the number of total MP and all MP subsets were higher than those in healthy controls $(p<0.05)$. Also, all MP subsets were significantly higher in patients with dcSSc compared to limited cutaneous SSc $(p<0.001)$. We also found that the number of PMP, EMP and MoMP were higher in patients who were positive against anti-topoisomerase I antibody than those negative $(p<0.01, p<0.05$ and $p<0.05$, respectively), whereas there were no significant differences in the numbers of each MP subset between the patients positive or negative against other SScspecific autoantibodies. In addition, the number of PMP and EMP were higher in patients with interstitial lung disease than those without $(p<0.05)$. Moreover, all MP subsets were significantly inversely correlated with the percentage of predicted forced vital capacity $(p<0.05, p<0.01$, and $p<0.01$, respectively).

Conclusions: These results suggest that MP subsets are associated with extended fibrotic phenotype in patients with SSc, and might be utilized as novel biomarkers.

References:

[1] Bayer $C$ et al. Nat Rev Rheumatol. 6:21-9, 2010

[2] Antonyak MA et al. Method Mol Biol 1165:147-73, 2014.

[3] Budoni M et al. Cell Transplant. 22:369-79, 2013.

Disclosure of Interest: None declared

DOI: 10.1136/annrheumdis-2017-eular.4275 